\title{
Reduced Left Executive Control Network Functional Connectivity Is Associated with Alcohol Use Disorders
}

\author{
Barbara J. Weiland, Amithrupa Sabbineni, Vince D. Calhoun, Robert C. Welsh, \\ Angela D. Bryan, Rex E. Jung, Andrew R. Mayer, and Kent E. Hutchison
}

\begin{abstract}
Background: Altered functional connectivity in critical networks has been associated with chronic alcohol abuse. In turn, changes in connectivity in executive control networks (ECNs) may undermine the ability to control alcohol consumption. It was hypothesized that network connectivity would be reduced in individuals with problematic alcohol use (ALC) compared with controls and that diminished network connectivity would be associated with greater failure to control drinking.

Methods: Resting-state functional magnetic resonance imaging was analyzed to identify 14 previously identified intrinsic connectivity networks (ICNs) using a priori regions of interest in cases ranging from binge drinkers to those with severe alcohol use disorder, as well as control subjects. Analyses tested for differences in network connectivity strength between 255 ALC cases and 87 age- and gendermatched controls. Further, structural equation analysis, using 383 ALC cases, tested whether functional connectivity strength mediated the relationship between years of regular drinking and alcohol problems.

Results: The age- and gender-matched analysis showed that ALC had significantly lower network connectivity strength than controls in the left executive control (LECN), basal ganglia, and primary visual networks. For all ALC, LECN connectivity strength is negatively correlated with failed control and alcohol disorder severity. Edges connecting parietal regions with dorsolateral prefrontal, middle frontal, and temporal regions within the LECN drove these relationships. A positive association between years of drinking and severity of alcohol problems was mediated by reduced ECN connectivity.

Conclusions: This study reports relationships between network strength and problematic alcohol use, suggesting that chronic drinking negatively impacts brain connectivity, specifically in the LECN. Altered functional connectivity, related to chronic alcohol abuse, may contribute to the etiology of alcohol dependence and relapse.

Key Words: Alcohol Dependence, Executive Control, Functional Connectivity, Functional Network, Resting State.
\end{abstract}

$\mathrm{T}$ HE CONSEQUENCES OF heavy alcohol use on brain structure have been studied extensively using imaging modalities to repeatedly demonstrate negative impacts from chronic use. For example, overall decreases in brain volume have been found to covary with alcohol intake (Harding et al., 1996), theorized to result from the neurotoxic effects

From the Department of Psychology and Neuroscience (BJW, AS, $A D B, K E H)$, University of Colorado Boulder, Boulder, Colorado; Department of Electrical and Computer Engineering (VDC), University of New Mexico, Albuquerque, New Mexico; The Mind Research Network (VDC, ADB, ARM, KEH), Albuquerque, New Mexico; Department of Radiology $(R C W)$, University of Michigan, Ann Arbor, Michigan; Department of Psychiatry ( $R C W)$, University of Michigan, Ann Arbor, Michigan; Department of Neurosurgery (REJ), University of New Mexico, Albuquerque, New Mexico; and Department of Neurology (ARM), University of New Mexico, Albuquerque, New Mexico.

Received for publication February 7, 2014; accepted May 27, 2014.

Reprint requests: Barbara J. Weiland, PhD, Department of Psychology and Neuroscience, University of Colorado Boulder University of Michigan, Campus Box 345, Boulder, CO 80309; Tel.: 303-492-9147; Fax: 303-492-2967; E-mail: barbara.weiland@colorado.edu

Copyright $(2014$ by the Research Society on Alcoholism.

DOI: 10.1111/acer.12505 of alcohol (Lishman, 1990). The frontal lobe and limbic systems, basal forebrain, and cerebellum have been shown to be particularly vulnerable to alcoholism-related damage (Dirksen et al., 2006; Kril et al., 1997; Oscar-Berman et al., 1997; Pfefferbaum et al., 1997; Sullivan et al., 2000). These regions play key roles in cognitive processes including executive functioning (Sullivan and Pfefferbaum, 2005), as well as mediating and reinforcing appetitive drive hypothesized to influence the inability to control alcohol consumption (Everitt and Robbins, 2005).

Further, functional connectivity analyses, which quantify the connections between brain regions based on temporal correlation (Wig et al., 2011), have shown that chronic alcohol use affects relationships among these important regions. For example, compared with healthy controls (HC), recently abstinent alcoholic patients showed reduced fronto-cerebellar functional connectivity derived from a motor task (Rogers et al., 2012) and reduced fronto-striatal connectivity during response inhibition (Courtney et al., 2013). Longterm abstinent alcoholic subjects show reduced resting-state synchrony between the nucleus accumbens (NAcc) and thalamus, caudate, postcentral, and parietal regions, yet 
increases between the NAcc and frontal regions. The authors suggested that reduced connectivity between decision-making regions with appetitive drive regions yet increased connectivity with inhibitory control regions may represent an adaptive mechanism which helps sustain abstinence (Camchong et al., 2013). Further, functional connectivity measured within multiple networks was lower in relapsing alcoholics compared with those who sustained abstinence (Beck et al., 2012; Camchong et al., 2012). These studies converge to support dysfunctional connectivity, associated with sustained alcohol use, as an underlying mechanism contributing to poor inhibitory control.

Understanding the effects of alcohol abuse on functional connectivity has important implications for the etiology and treatment of alcohol dependence. Given that only a few studies have examined these effects, we sought to examine the association between problem alcohol use and functional connectivity in individuals ranging from binge drinkers to those with severe alcohol use disorder (ALC). As the studies above demonstrated changes in multiple brain networks, we chose to utilize the functionally defined intrinsic connectivity networks (ICNs) defined by Shirer and colleagues (2012). These 14 ICNs are comprised of 90 distinct regions of interest (ROIs), or network nodes, that encompass the majority of the cortical and subcortical gray matter (Shirer et al., 2012) and can be mapped to canonical functions: auditory; primary and higher visual; language; sensorimotor (SM); anterior and posterior salience; basal ganglia (BG); dorsal, ventral, and precuneus default mode; and bilateral executive control systems (Beckmann et al., 2005; Chakravarthy et al., 2010; Damoiseaux et al., 2006; Greicius et al., 2003; Hampson et al., 2006; Kiviniemi et al., 2009; Seeley et al., 2007; Smith et al., 2009). As long-term heavy drinking has been shown to have detrimental effects on cognition and executive function (Goldstein et al., 2004; Parsons and Nixon, 1993; Pitel et al., 2007; Sullivan et al., 2000), we were particularly interested in networks involved in these functions, specifically the right and left executive control network (R/L ECN). We hypothesized that ALC subjects would have reduced connectivity strength in the both ECNs and that lower ECN connectivity strength would be correlated with disease severity.

As studies have shown that aging has an impact on functional connectivity (Allen et al., 2011; Andrews-Hanna et al., 2007; Damoiseaux et al., 2008; Tomasi and Volkow, 2012), we first compared an age-matched subset of ALC subjects with a group of $\mathrm{HC}$ to examine connectivity differences associated with problematic alcohol use. We expected that the ALC cases would have reduced resting-state synchrony within ECNs implicated in addiction, but also tested whether differences compared with controls might be found globally within all ICNs. We also posited that reduced within-network functional connectivity in the ECNs may be the neurobiological link between the neurotoxic effects of chronic alcohol exposure and problem alcohol use, having a mediating role on this relationship. To test this hypothe- sis, we used a structural equation model to test the indirect effect of alcohol exposure on problem alcohol use via network connectivity strength.

\section{MATERIALS AND METHODS}

\section{Participants}

Four hundred and twenty-two individuals with ALC and 97 controls were recruited from the greater Albuquerque metropolitan region through advertisements in local print, online media, and radio advertisements. Subjects were paid $\$ 120$ for participation in both the questionnaire and neuroimaging sessions. ALC participants had to report binge drinking ( 5 or more drinks per drinking occasion for men, 4 or more for women) at least 5 times in the past month with exclusionary criteria of previous brain injury or loss of consciousness for more than 5 minutes, a history of severe alcohol withdrawal, or a positive pregnancy test. Additional exclusionary criteria for the $\mathrm{HC}$ subjects included a history of neurological disorder, mental retardation, lifetime history of dependence or use within the last 12 months of PCP, amphetamines or cocaine, current or past psychiatric disorder (with the exception of 1 lifetime depressive episode), antidepressant use within the last 6 months, and lifetime antidepressant use of more than 1 year. Subjects were required to stop drinking 24 hours prior and pass a breathalyzer prior to participation. Individuals with excessive motion $(>2 \mathrm{~mm}$ translational or 0.035 radians rotational movement, $n=39 \mathrm{ALC}, n=10 \mathrm{HC}$ ) or technical problems (wrong acquisition plane, $n=1$ ALC) were excluded. The final full ALC sample included 383 subjects with the following comorbid substance use within the past 60 days (No/Yes/Unknown): Cigarettes, 105/278; Marijuana, 213/146/24; other substances, 326/33/24 (cocaine-11; methamphetamine-3; hallucinogens-5; opiates-3; 2 or more of these substances-11). Written informed consent, approved by the University of New Mexico Human Research Committee, was obtained from all participants.

To select the age- and gender-matched subsample of ALC subjects, all ALC subjects less than the mean age (25.6 years) of the $\mathrm{HC}$ group were included. The remainder of the ALC subjects who were younger than the maximum age (53.3 years) of the HC group were assigned random numbers and sequentially added to the ALC subsample until the 2 groups (ALC, $n=255$; HC, $n=87$ ) did not differ by age or gender.

\section{Measures}

ALC participants were assessed with the Alcohol Use Disorders Identification Test (AUDIT; scores $>8$ are indicative of hazardous drinking/dependence (Saunders et al., 1993), the Failed Control subscale of the Impaired Control Scale (ICS-FC; evaluating failure of attempts to control drinking in the last 6 months (Heather et al., 1998), and a questionnaire on drinking which including the question, "For how many years have you been drinking regularly?" to determine years of regular drinking (YRD).

\section{Image Acquisition}

Whole brain resting-state functional magnetic resonance imaging was performed on a 3-T SiemensTrio (Erlangen, Germany) scanner with a 12-channel radio frequency coil. In the scanner, tape was placed across the participants' forehead to serve as feedback for movement reduction. $T_{2}{ }^{*}$-weighted functional images were acquired using a gradient-echo echo-planer imaging sequence: TE $=29 \mathrm{~ms}$, $\mathrm{TR}=2$ seconds, flip angle $=75^{\circ}$, slice thickness $=3.5 \mathrm{~mm}$, slice gap $=1.05 \mathrm{~mm}$, field of view $=240 \mathrm{~mm}, 64 \times 64$ matrix, voxel 
size $=3.75 \mathrm{~mm} \times 3.75 \mathrm{~mm} \times 3.5 \mathrm{~mm}$. Resting-state scans were 5 minutes in duration. Subjects were instructed to keep their eyes open and fixate on a cross. High-resolution $\mathrm{T}_{1}$-weighted structural image were acquired with a 5-echo multiecho MPRAGE sequence: $\mathrm{TE}=1.64,3.5,5.36,7.22$, and $9.08 \mathrm{~ms}, \mathrm{TR}=2.53$ seconds, $\mathrm{TI}=1.2$ seconds, flip angle $=7$, excitations $=1$, slice thickness $=1 \mathrm{~mm}$, field of view $=256 \mathrm{~mm}$, resolution $=$ $256 \times 256 \times 176$, voxel size $1 \times 1 \times 1 \mathrm{~mm}$, pixel bandwidth $=$ $650 \mathrm{~Hz}$.

\section{Image Preprocessing}

Functional images were preprocessed using an automated pipeline based around SPM 5 (http://www.fil.ion.ucl.ac.uk/spm/software/spm5) including realignment, slice-timing correction, spatial normalization to Montreal Neurological Institute (MNI) space, reslicing, and smoothing with a 10-mm full-width half-max Gaussian kernel (Scott et al., 2011). Time series of cerebrospinal fluid and white matter fluctuations, motion parameters, and first derivative of motion parameters were progressively regressed from the time-series data followed by band-pass filtering $(0.01-0.1 \mathrm{~Hz})$ using in-house scripts (Welsh et al., 2010). For additional exploration of data processing, including motion exclusion and despiking, see Supplemental Methods.

\section{Network Functional Connectivity Analysis}

The 14 ICNs were identified using publicly available, functionally defined ROIs (Shirer et al., 2012; Fig. 1). Functional connections between nodes, defined as edges, were calculated, creating a correlation matrix between the time series of all nodes within each network for each subject, again using in-house scripts (Welsh et al., 2010). A Fisher $r$-to- $z$ transformation was applied to yield $z$-scores for use in subsequent analyses. Connectivity strength is a global measure of connectivity calculated as the mean of all pairwise correlations between nodes within each network (Lynall et al., 2010). As motion has been shown to influence functional connectivity (Van Dijk et al., 2012), the framewise displacement (FD) (Power et al., 2012) for each subject was calculated across the entire resting state run from the image motion parameters for use as a covariate. For additional seed-based analyses, validating these networks for the ALC sample, see Supplemental Methods.

\section{Data Analyses}

SPSS Statistical Software version 18 (Chicago, IL) was used to perform multivariate analysis of variance (MANCOVA) including FD as a covariate to determine whether the matched HC and ALC groups differed on connectivity strength including all 14 networks.

For networks identified as significant in the univariate analysis of the MANCOVA, relationships between network strength with AUDIT and ICS-FC scores were assessed for all ALC subjects.

\section{Model to Test the Indirect Effect of Alcohol Exposure on Alcohol} Problems Through Network Connectivity Strength

To test the indirect effect of alcohol exposure on alcohol problems via ECN connectivity strength, we tested the model in Fig. 2 for the entire ALC sample in EQS Version 6.1 (Bentler and Wu, 2003). The dependent measure is a latent variable defined as alcohol problems with AUDIT and ICS-FC serving as indicators. As age

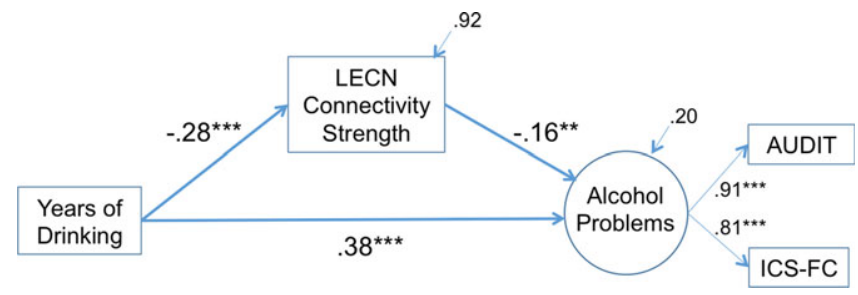

Fig. 2. Model of network connectivity as a mediator. A significant mediation of the effect of years drinking on disorder severity through network connectivity was found $(z=-3.07, p<0.01)$. LECN, left executive control network; AUDIT, Alcohol Use Disorders Identification Test; ICS-FC, Failed Control subscale of Impaired Control Scale. $* * p<0.01, * * * p<0.001$. Yuan-Bentler $\chi^{2}(1)=0.869, p=0.35, \mathrm{CFI}=1.0, \mathrm{RMSEA}=0.00$.

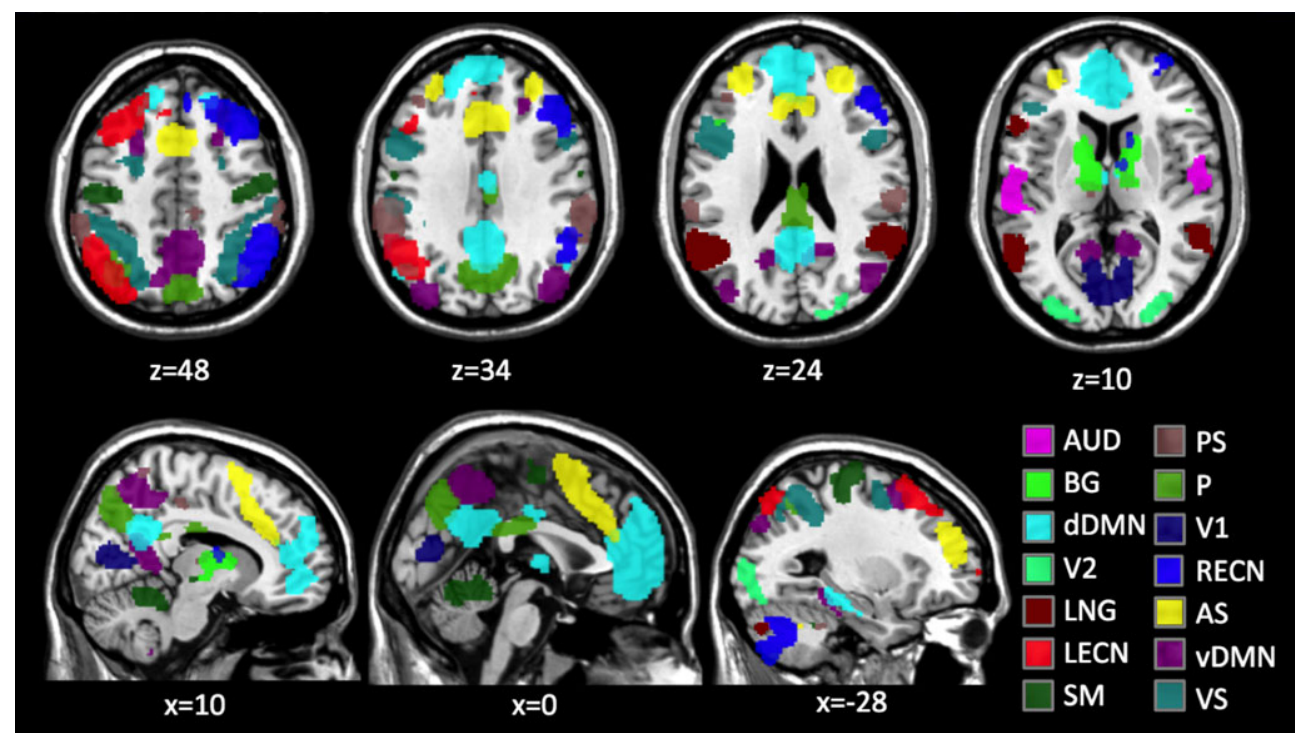

Fig. 1. Fourteen intrinsic connectivity networks (ICNs). The ICNs are comprised of 90 distinct regions of interest or nodes encompassing the majority of the cortical and subcortical gray matter that were downloaded from Stanford's FIND Laboratory (Shirer et al., 2012) and include: auditory; primary and higher visual; language; sensorimotor; anterior and posterior salience; basal ganglia; dorsal, ventral and precuneus default mode; and bilateral executive control systems. 
and YRD were highly correlated in the ALC sample $(r=0.727$, $p<0.001$ ), we chose to use YRD as the sole exogenous variable in the model.

\section{RESULTS}

\section{Demographic and Psychometric Measures}

Table 1 shows sample characteristics for all groups. The $\mathrm{HC}$ and ALC subset were well matched on age, $t=1.60$, $p=0.110$, and gender, $\chi^{2}=0.088, p=0.766$.

\section{Group Network Connectivity Analysis}

The MANCOVA revealed a significant effect of group on network connectivity strength, $F(14, \quad 326)=2.910$,

Table 1. Subject Characteristics for Control and Alcohol Use Disorder Subjects

\begin{tabular}{|c|c|c|c|}
\hline & Controls & $\begin{array}{l}\text { Age-matched } \\
\text { ALC }\end{array}$ & All ALC \\
\hline $\mathrm{N}$ & 87 & 255 & 383 \\
\hline Males:Females & $59: 37$ & 162:104 & $257: 150$ \\
\hline Age & $25.8(8.3)$ & $26.9(6.9)$ & $31.1(9.3)$ \\
\hline Range & $21.0-53.3$ & $21.0-53.0$ & 21.0 to 56.0 \\
\hline $\begin{array}{l}\text { Years of regular } \\
\text { drinking }\end{array}$ & NA & $7.8(6.7)$ & $11.9(9.3)$ \\
\hline ICS-FC & NA & $14.8(9.1)$ & $16.8(9.6)$ \\
\hline AUDIT & NA & $14.9(7.0)$ & $16.3(7.9)$ \\
\hline \multicolumn{4}{|l|}{ Ethnicity } \\
\hline Caucasian & 44 & 115 & 175 \\
\hline African American & 1 & 5 & 8 \\
\hline $\begin{array}{l}\text { Asian/Native } \\
\text { Hawailan }\end{array}$ & 3 & 3 & 4 \\
\hline Latino & 18 & 53 & 82 \\
\hline Native American & 3 & 26 & 35 \\
\hline Mixed & 10 & 30 & 48 \\
\hline Unknown & 8 & 23 & 31 \\
\hline \multicolumn{4}{|l|}{ Network connectivity } \\
\hline LECN & $0.46(0.14)^{\star}$ & $0.41(0.14)$ & $0.38(0.14)$ \\
\hline RECN & $0.45(0.12)$ & $0.44(0.13)$ & $0.42(0.13)$ \\
\hline AS & $0.39(0.10)$ & $0.41(0.12)$ & $0.40(0.12)$ \\
\hline PS & $0.22(0.07)$ & $0.21(0.07)$ & $0.21(0.07)$ \\
\hline dDMN & $0.38(0.11)$ & $0.40(0.11)$ & $0.38(0.12)$ \\
\hline vDM & $0.37(0.09)$ & $0.39(0.11)$ & $0.38(0.11)$ \\
\hline$P$ & $0.48(0.14)$ & $0.50(0.14)$ & $0.49(0.14)$ \\
\hline$B G$ & $0.21(0.09)^{*}$ & $0.17(0.09)$ & $0.17(0.09)$ \\
\hline SM & $0.32(0.12)^{\star \star}$ & $0.29(0.11)$ & $0.30(0.12)$ \\
\hline AUD & $0.48(0.17)$ & $0.46(0.15)$ & $0.46(0.15)$ \\
\hline LNG & $0.35(0.11)$ & $0.35(0.11)$ & $0.34(0.11)$ \\
\hline PV & $0.20(0.18)^{\star}$ & $0.14(0.17)$ & $0.14(0.18)$ \\
\hline $\mathrm{HV}$ & $1.26(0.33)$ & $1.29(0.29)$ & $1.28(0.29)$ \\
\hline VS & $0.41(0.11)$ & $0.40(0.12)$ & $0.39(0.12)$ \\
\hline $\begin{array}{l}\text { Framewise } \\
\text { displacement }\end{array}$ & $0.17(0.08)$ & $0.19(0.09)$ & $0.21(0.10)$ \\
\hline
\end{tabular}

Data presented as mean (standard deviation) where applicable.

ALC, individuals with problematic alcohol use; NA, not applicable; ICSFC, Impaired Control Scale, Failed Control subscale; AUDIT, Alcohol Use Disorder Identification Test; LECN, left executive control network; RECN, right executive control network; AS, anterior salience; PS, posterior salience; dDMN, dorsal default mode; vDM, ventral default mode; $P$, precuneus; BG, basal ganglia; SM, sensorimotor; AUD, auditory; LNG, language; PV, primary visual; HV, high visual; VS, visuospatial.

${ }^{*}$ Significant group difference, $p=0.024$.

${ }^{* *}$ Significant group difference, $p \leq 0.001$. $p<0.001$, partial $\eta^{2}=0.111$; univariate tests showed that the $\mathrm{HC}$ and age- and gender-matched ALC groups differed on connectivity strength for 4 networks (LECN: $F=5.348$, $p=0.021 ; \quad \mathrm{SM}: F=5.177, \quad p=0.024 ; \quad$ BG: $F=14.234$, $p<0.001$; primary visual (PV): $F=5.171, p=0.024)$ indicating that chronic alcohol use, not age was driving these network differences (Table 2, Figs 3 and 4).

For all ALC, LECN strength was negatively correlated with both ICS-FC and AUDIT scores (Table 3) remaining significant when controlling for $\mathrm{FD}$, gender, and age $(r=-0.125, p=0.015$ and $r=-0.158, p=0.002$, respectively), which suggests that motion, gender, nor age were driving the results. To further ensure that age was not driving these effects, the sample was split at 40 years, finding the same negative relationships between LECN connectivity and ICS-FC and AUDIT within both groups: young ALC $(n=307$, age $=27.2$ [5.1]): $r=-0.161, \quad p=0.005$ and $r=-0.177, p=0.002$, respectively; old ALC $(n=76$, age $=46.7[4.5]): r=-0.299, p=0.009$ and $r=-0.264$, $p=0.021$, respectively. Connectivity strength of the other networks identified in the MANCOVA was not related to either ICS-FC or AUDIT.

Further exploration within the LECN was carried out by evaluating relationships between the behavioral measures and each edge $z$-score again controlling for motion. The significance threshold for edge correlations was set as $p=0.05$ / $n$, where $n=$ number of edges within a network; for the LECN, which has 30 edges, the significance threshold was $p=0.0017$. For all ALC, within the LECN, negative correlations with ICS-FC and/or AUDIT were found with edges between the dorsolateral prefrontal cortex (DLPFC) and parietal lobe (PAR), middle frontal gyrus (MFG), and temporal gyrus (TL), and between TL and both MFG and PAR (Fig. 5 and Table 4).

\section{Mediation Model}

In the mediation model, which included the entire ALC sample, robust estimation with maximum likelihood estimation of missing data was utilized. Thus, we present the robust fit statistics and significance of the parameters evaluated with robust standard errors. The mediation model produced an excellent fit to the data (Fig. 2). There were significant paths from YRD to LECN connectivity strength ( $B=-0.43, p<0.001)$, indicating that more years of regular drinking were associated with weaker connectivity. There was also a significant path from LECN connectivity strength to alcohol problems $(B=-0.24, p<0.001)$, indicating higher connectivity strength was associated with less problematic drinking. The indirect effect of years drinking on alcohol problems was significant $(z=-2.86, p<0.01)$, indicating a significant mediated effect. However, mediation was not complete, as there was a remaining significant direct effect of years drinking on problematic drinking $(B=0.31, p<0.001)$. 

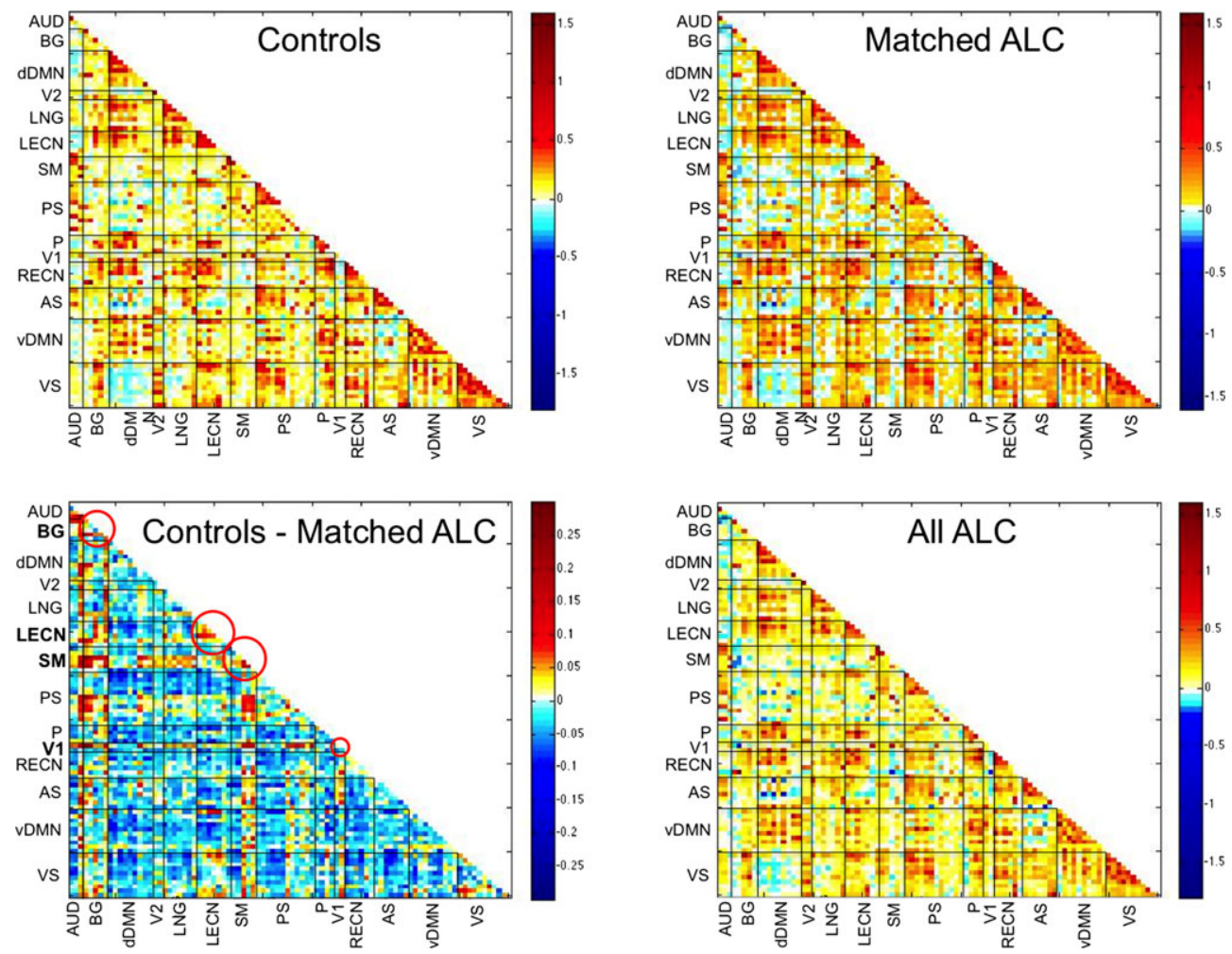

Fig. 3. Correlation matrices across all 14 intrinsic connectivity networks (ICNs). Average correlation matrices of functional connectivity for all ICNs by group: healthy controls $(n=87)$; age- and gender-matched ALC subset $(n=255)$; and all ALC $(n=383)$. The difference matrix, controls-matched ALC, shows the networks that differed between the matched groups with the ALC groups having lower network functional connectivity strength. ALC, individuals with problematic alcohol use.

\section{DISCUSSION}

Using a large sample with a range of alcohol problems, this study investigated the relationship between chronic alcohol abuse and resting-state functional connectivity of brain networks critically involved in regulation and control. The age- and gender-matched subset of ALC subjects had significantly lower network strength in the LECN, SM, BG, and PV than controls. Further, for the entire sample of ALC subjects, LECN connectivity strength was negatively associated with measures of hazardous drinking and loss of control over alcohol consumption. Within the LECN, we identified key nodes significantly associated with disease severity. We then tested a model to show that LECN connectivity strength mediates the relationship between chronic alcohol exposure and problematic alcohol use. Given our sample size was an order of magnitude greater than previous studies, the current study highlights the importance of the LECN in the addiction cycle. Our results suggest that detrimental degradation of pathways within the left executive system may be a critical neurobiological mechanism through which chronic alcohol use impairs individuals' ability to control or discontinue use.

Neuroimaging research supports the existence of multiple large-scale connected networks with distinct functional and behavioral domains (Fair et al., 2007; Laird et al., 2011), including complex cognitive systems involved in multilevel functions including decision making, inhibition, and response control. Of particular interest, poor cognitive control has been suggested to be involved in the addiction cycle, particularly with respect to maintaining abstinence. These dysfunctions in control are thought to be further negatively impaired with chronic alcohol use (see reviews including Lyvers, 2000; Moselhy et al., 2001). While these "executive functions" have historically been associated with the "frontal lobe" (Baddeley et al., 1997; Miyake et al., 2000), a network, or systems, approach posits that the coordinated interaction of multiple brain regions is a critical component of executive function. Using a functional approach, bilateral ECNs have been identified that include regions in the PAR, DLPFC, the MFG, and contralateral cerebellar areas (Shirer et al., 2012), which we queried for this study.

As hypothesized, we found that network strength, in LECN, though not in the RECN, was lower in ALC than $\mathrm{HC}$ and negatively correlated with disorder severity. Within the LECN, we examined the edges, or the pairwise temporal correlations between ROIs to identify the nodes that seemed to drive this relationship. We found negative associations between both failed control and AUDIT scores and the connectivity between the PAR, DLPFC, MFG, and caudate nodes, suggesting poor function of this control network. This is consistent with studies that have reported negative associations between white matter integrity in tracts connecting 

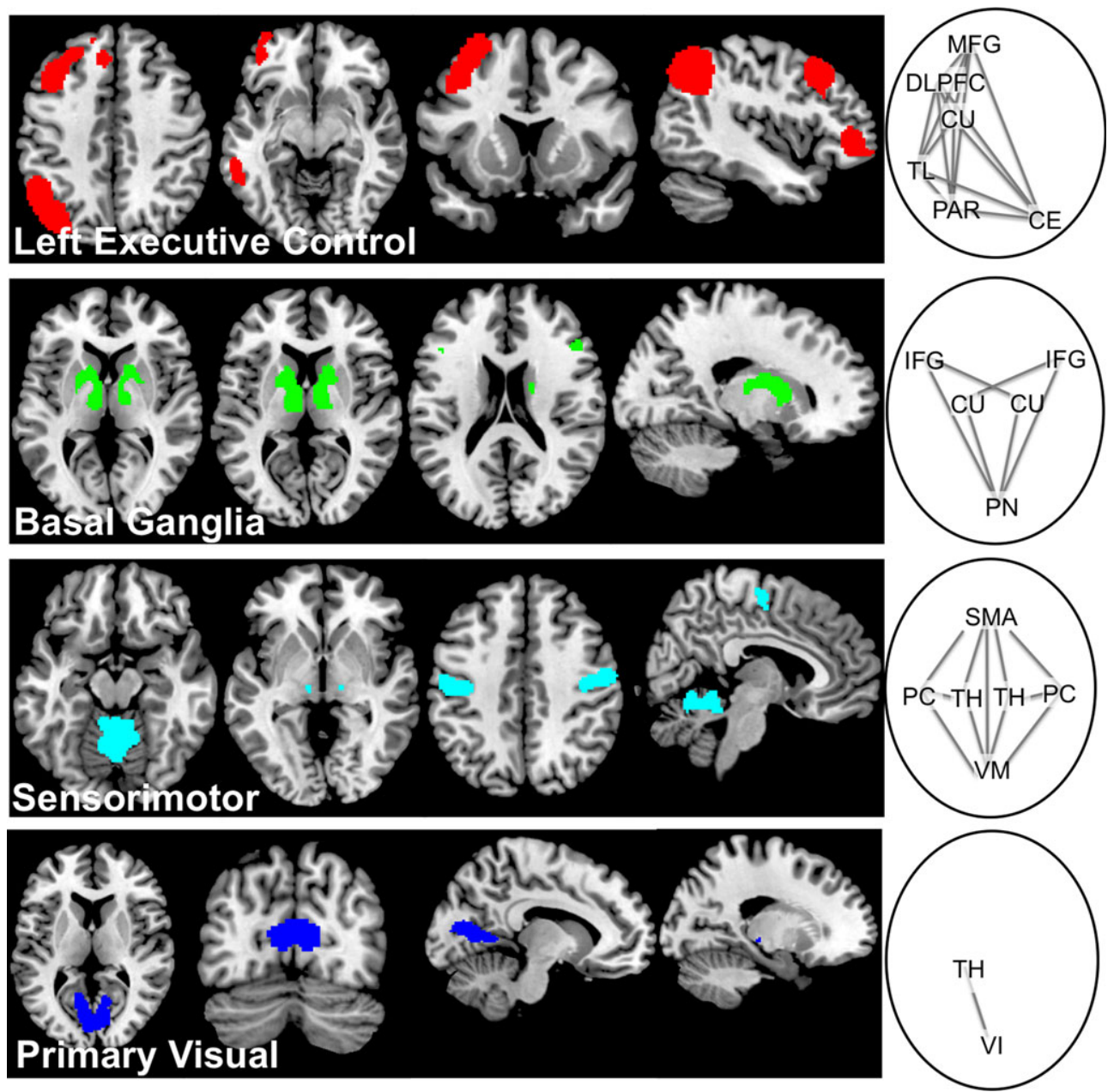

Fig. 4. Networks different between individuals with problematic alcohol use (ALC) and controls. Regions of interest defining the networks with significantly lower connectivity strength in age- and gender-matched ALC subjects $(n=255)$ and controls $(n=87)$ include the left executive control network (LECN), basal ganglia (BG), sensorimotor (SM), and primary visual (PV) networks. Table 2 details the acronyms of the network nodes displayed in the right panel.

frontal and parietal regions and substance use (Pfefferbaum et al., 2009; Schulte et al., 2010), validating the important relationship between these structures and impaired control in ALC. Of interest, a recent study of stimulant-dependent individuals, a majority of whom was also dependent on alcohol, found a lateralized differentiation of the ECNs. As resting-state amplitude in the left DLPFC correlated with approach behaviors, the authors suggested that the LECN may be involved in maintaining a bias toward seeking and taking drugs, rather than representing a failure of executive control (Krmpotich et al., 2013). Our results, however, would suggest that poor communication between critical nodes within the LECN might, in fact, represent such a failure.

Failed control over alcohol consumption, which has been correlated with measures of alcohol dependence and negatively predictive of treatment outcome (Heather et al., 1998), was found here to negatively relate to LECN connectivity strength, suggesting an underlying mechanism for control dysfunction. Specifically, deficits in the connectivity integrity between key structures may be involved in loss of control over drinking. A primary structure that showed reduced edge connectivity was the DLPFC, a region associated with topdown attentional focus thought to be involved in cognitive control over memory representations. This control is hypothesized to be influenced by emotional information and impacts including that from the MFG, which is implicated in mood awareness and regulation; the MFG was another primary node with poor edge connectivity. We also identified poor connectivity with the PAR node which is suggested to be involved in the complementary roles of sustaining attention and responding to salient stimuli (Singh-Curry and Husain, 2009). Thus, reduced connectivity between these critical regions, across our large ALC sample, suggests inefficient innetwork communication of goals and priorities, potentially contributing to poor behavioral control.

Our results, finding differences between ALC and controls in multiple networks, are in agreement with work showing resting-state connectivity altered with other substance use from cocaine (Gu et al., 2010; Krmpotich et al., 2013), 
Table 2. Definition of Nodes for Networks Different Between ALC Subjects $(n=255)$ and Controls $(n=87)$

\begin{tabular}{|c|c|c|}
\hline Network & Area & Abbreviation \\
\hline \multirow[t]{6}{*}{$\begin{array}{l}\text { Left executive } \\
\text { control }\end{array}$} & $\begin{array}{l}\text { 1. Left Middle Frontal Gyrus/Superior } \\
\text { Frontal Gyrus }\end{array}$ & DLPFC \\
\hline & $\begin{array}{l}\text { 2. Left Inferior Frontal Gyrus/Orbitofrontal } \\
\text { Gyrus }\end{array}$ & MFG \\
\hline & $\begin{array}{l}\text { 3. Left Superior Parietal Gyrus/Inferior } \\
\text { Gyrus/Precuneus/Angular Gyrus }\end{array}$ & PAR \\
\hline & $\begin{array}{l}\text { 4. Left Inferior Temporal Gyrus/ } \\
\text { Middle Temporal Gyrus }\end{array}$ & TL \\
\hline & 5. Right Crus I/Crus II/Lobule VI & CE \\
\hline & 6. Left Thalamus/Caudate & $\mathrm{CU}$ \\
\hline \multirow[t]{5}{*}{ Basal ganglia } & $\begin{array}{l}\text { 1. Left Caudate/Putamen/Pallidum/ } \\
\text { Thalamus }\end{array}$ & $\mathrm{CU}$ \\
\hline & $\begin{array}{l}\text { 2. Right Caudate/Putamen/Pallidum/ } \\
\text { Thalamus }\end{array}$ & $\mathrm{CU}$ \\
\hline & 3. Left Inferior Frontal Gyrus & IFG \\
\hline & 4. Right Inferior Frontal Gyrus & IFG \\
\hline & 5. Pons & PN \\
\hline \multirow[t]{7}{*}{ Sensorimotor } & 1. Left Pre/Postcentral Gyri & PC \\
\hline & 2. Right Pre/Postcentral Gyri & $\mathrm{PC}$ \\
\hline & 3. Supplementary Motor Area & SMA \\
\hline & 4. Right Middle Frontal Gyrus & MFG \\
\hline & 5. Left Thalamus & $\mathrm{TH}$ \\
\hline & 6. Right Thalamus & $\mathrm{TH}$ \\
\hline & 7. Vermis & VM \\
\hline \multirow[t]{2}{*}{ Primary visual } & 1. Primary Visual & V1 \\
\hline & 2. Left Thalamus & $\mathrm{TH}$ \\
\hline
\end{tabular}

ALC, individuals with problematic alcohol use.

Table 3. Correlations of Network Connectivity Strengths with Measures of Alcohol Disorder Severity $(n=383)$

\begin{tabular}{|c|c|c|c|c|}
\hline \multirow[b]{2}{*}{ Network strength } & \multicolumn{2}{|c|}{ ICS-FC } & \multicolumn{2}{|c|}{ AUDIT } \\
\hline & $r$ & $p$ & $r$ & $p$ \\
\hline Left executive control & -0.250 & $<0.001$ & -0.254 & $<0.001$ \\
\hline Basal ganglia & -0.007 & 0.893 & 0.005 & 0.929 \\
\hline Sensorimotor & 0.001 & 0.988 & -0.047 & 0.355 \\
\hline Primary visual & -0.003 & 0.989 & 0.000 & 0.996 \\
\hline
\end{tabular}

ICS-FC, Impaired Control Scale, Failed Control subscale; AUDIT, Alcohol Use Disorder Identification Test.

Bold indicates significant correlation.

heroin (Upadhyay et al., 2010), and prescription opioids (Upadhyay et al., 2010) to alcohol (Camchong et al., 2013; Chanraud et al., 2011). The lower resting-state connectivity we report in the SM network also agrees with task-related results finding reduced connectivity between premotor and cerebellar regions in alcoholics and is consistent with neuropathological deficits associated with alcoholism (Rogers et al., 2012). However, Chanraud and colleagues (2011) reported that controls $(n=15)$ had more positive restingstate connectivity within the default mode network than ALC subjects $(n=15)$, which we did not find. Further, Camchong and colleagues (2012) found that relapsing alcoholics $(n=29)$ had lower connectivity not only within the ECNs, but also within reward, visual, and salience networks, than abstainers $(n=40)$, where we did not find significant effects, possibly attributable to our large sample of subjects with greater variance of disorder severity. Our study also did not
Table 4. Edges with Significant Relationships with Failed Control and Alcohol Use Severity Measures Controlling for Motion for $n=383$ ALC Subjects

\begin{tabular}{lccccc}
\hline & \multicolumn{2}{c}{ ICS-FC } & & \multicolumn{2}{c}{ AUDIT } \\
\cline { 2 - 3 } \cline { 5 - 6 } $\begin{array}{l}\text { Network } \\
\text { Edges between brain nodes }\end{array}$ & $r$ & $p$ & & $r$ & $p$ \\
\hline LECN & & & & & \\
L DLPFC-L MFG & -0.135 & 0.00828 & & -0.186 & $\mathbf{0 . 0 0 0 2 6}$ \\
L DLPFC-L PAR & -0.213 & $\mathbf{0 . 0 0 0 0 3}$ & & -0.215 & $\mathbf{0 . 0 0 0 0 2}$ \\
L DLPFC-L TL & -0.185 & $\mathbf{0 . 0 0 0 2 9}$ & & -0.215 & $\mathbf{0 . 0 0 0 0 3}$ \\
L MFG-L TL & -0.130 & 0.01116 & & -0.163 & $\mathbf{0 . 0 0 1 4 2}$ \\
L MFG-CE & -0.126 & 0.01399 & & -0.140 & $\mathbf{0 . 0 0 6 2 3}$ \\
L PAR-L TL & -0.176 & $\mathbf{0 . 0 0 0 5 8}$ & -0.194 & $\mathbf{0 . 0 0 0 1 5}$ \\
\hline
\end{tabular}

ALC, individuals with problematic alcohol use; ICS-FC, Impaired Control Scale, Failed Control subscale; AUDIT, Alcohol Use Disorder Identification Test; LECN, left executive control network; L, left; R, right; DLPFC, dorsolateral prefrontal cortex; MFG, middle frontal gyrus; PAR, parietal cortex; TL, temporal lobe; CE, cerebellum.

Bold indicates edge meets corrected significance threshold within the specified network as detailed in text.

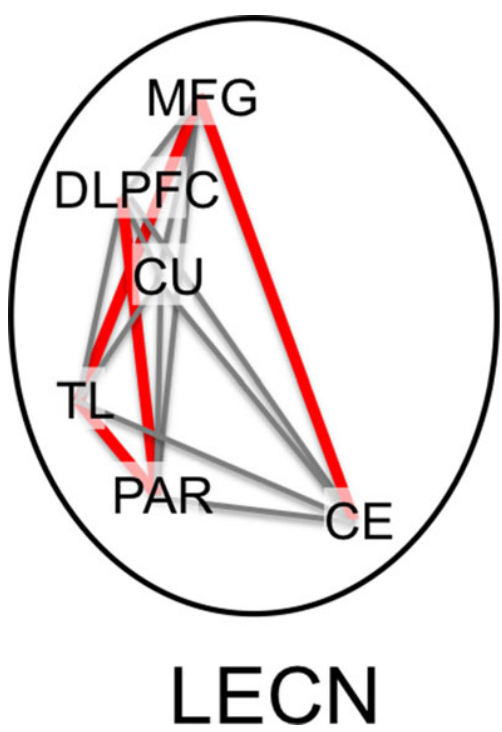

Fig. 5. Edges associated with alcohol problems. Depiction of edges within networks significantly correlated with failed control of alcohol consumption and/or alcohol use disorder severity within the left executive control network (LECN). Nodes between these edges include the left middle frontal gyrus (MFG), dorsolateral prefrontal cortex (DLPFC), temporal lobe $(\mathrm{TL})$, and right cerebellum (CE).

include follow-up data for the evaluation of relapse, however, given the finding that measures of severity were associated with degradation of network integrity only within the LECN across 383 ALC subjects, the negative impact of chronic alcohol use on this control system may be critically important in the addiction cycle. As task-related fronto-striatal connectivity reductions in abstinent alcoholics have been related to impairments in learning, as well as magnitude of alcohol craving (Park et al., 2010), perhaps deficits in resting-state LECN functional connectivity, representing reduced communication and cooperation between control system nodes, would also be predictive of cognitive decline. This should be studied in the future work, particularly in light of a recent report finding no cognitive deficits in 
treatment naïve alcoholics in contrast with consistently reported deficits in chronic, in-treatment alcoholics (Smith and Fein, 2010).

Several limitations of this study should be noted. The connectivity analysis here reveals interregional correlations but not information regarding causal or directional relationships between nodes, or whether other brain regions may drive these relationships. To identify ICNs, we used a publicly available atlas of functional ROIs (Shirer et al., 2012). This approach defined the networks of interest based on an independent sample to allow for unbiased evaluation of differences between our cases and controls. Network definition is an ongoing question in the field, and the use of another atlas, or of data driven ROIs, may be evaluated as a focus of the future work. In addition, this study is cross-sectional and observational, thus we are unable to assess causation, and it is possible that other models would be an equally good fit to the data (cf., MacCallum et al., 1993). Further, given the high correlation between the length of chronic alcohol use and age, we are unable to determine the independent effects of these variables in this study. And despite the correlation we report between LECN and disorder severity, the effect size is moderate. Other factors, likely genetic and environmental, are undoubtedly contributing to subjects' alcohol problems. Finally, it is possible that the ALC subjects in our study may have had preexisting differences in functional connectivity that contributed to vulnerability to their disorder rather than the lower network strengths being the consequences of chronic use. Longitudinal imaging studies, as well as studies of at-risk populations, are needed to further investigate this possibility.

The current study reports negative relationships between functional connectivity strength in the LECN, duration of chronic alcohol use, and disorder severity that may represent how such alcohol use negatively impacts brain function. Deterioration in functional connectivity strength in the LECN involved in cognitive control, decision-making and regulatory processes, may represent less efficient communication and function within this important network. These results suggest that altered functional connectivity related to problematic alcohol use may contribute to the underlying pathology of addiction, particularly with increasing longevity of disease.

\section{ACKNOWLEDGMENTS}

This work was supported by NIH grants to BJW from the National Institute on Drug Abuse (NIDA) K01 DA031755; to VDC from the National Institute of Biomedical Imaging and Bioengineering (NIBIB) 2R01EB000840; to RCW from National Institute of Neurological Disorders and Stroke (NINDS) R01052514; to KEH from NIDA R01DA025074 and the National Institute on Alcohol Abuse and Alcoholism (NIAAA) R01AA012238; a Brain and Behavior Foundation (NARSAD) Young Investigator grant to BJW; and a John Templeton Foundation grant to REJ. The authors report no biomedical financial interests or potential conflicts of interests. We acknowledge the contribution of Tiffany J. Callahan and Jameson P. Owens in quality control of data.

\section{REFERENCES}

Allen EA, Erhardt EB, Damaraju E, Gruner W, Segall JM, Silva RF, Havlicek M, Rachakonda S, Fries J, Kalyanam R, Michael AM, Caprihan A, Turner JA, Eichele T, Adelsheim S, Bryan AD, Bustillo J, Clark VP, Feldstein Ewing SW, Filbey F, Ford CC, Hutchison K, Jung RE, Kiehl KA, Kodituwakku P, Komesu YM, Mayer AR, Pearlson GD, Phillips JP, Sadek JR, Stevens M, Teuscher U, Thoma RJ, Calhoun VD (2011) A baseline for the multivariate comparison of resting state networks. Front Syst Neurosci 5: 1-23.

Andrews-Hanna JR, Snyder AZ, Vincent JL, Lustig C, Head D, Raichle ME, Buckner RL (2007) Disruption of large-scale brain systems in advanced aging. Neuron 56:924-935.

Baddeley A, Della Sala S, Papagno C, Spinnler H (1997) Dual-task performance in dysexecutive and nondysexecutive patients with a frontal lesion. Neuropsychology 11:187-194.

Beck A, Wüstenberg T, Genauck A, Wrase J, Schlagenhauf F, Smolka MN, Mann K, Heinz A (2012) Effect of brain structure, brain function, and brain connectivity on relapse in alcohol-dependent patients. Arch Gen Psychiatry 69:842-852.

Beckmann CF, DeLuca M, Devlin JT, Smith SM (2005) Investigations into resting-state connectivity using independent component analysis. Philos Trans R Soc Lond B Biol Sci 360:1001-1013.

Bentler PM, Wu EJ (2003) EQS 6.1 for Windows Beta Build 65 [Computer Software] [Computer Program]. Multivariate Software, Encino, CA.

Camchong J, Stenger A, Fein G (2012) Resting-state synchrony during early alcohol abstinence can predict subsequent relapse. Cereb Cortex 23: 20862099.

Camchong J, Stenger A, Fein G (2013) Resting-state synchrony in long-term abstinent alcoholics. Alcohol Clin Exp Res 37:75-85.

Chakravarthy VS, Joseph D, Bapi R (2010) What do the basal ganglia do? A modeling perspective. Biol Cybern 103:237-253.

Chanraud S, Pitel A-L, Pfefferbaum A, Sullivan EV (2011) Disruption of functional connectivity of the default-mode network in alcoholism. Cereb Cortex 21:2272-2281.

Courtney KE, Ghahremani DG, Ray LA (2013) Fronto-striatal functional connectivity during response inhibition in alcohol dependence. Addict Biol 18:593-604.

Damoiseaux JS, Beckmann CF, Arigita EJS, Barkhof F, Scheltens P, Stam CJ, Smith SM, Rombouts SARB (2008) Reduced resting-state brain activity in the "default network" in normal aging. Cereb Cortex 18:1856-1864.

Damoiseaux JS, Rombouts SA, Barkhof F, Scheltens P, Stam CJ, Smith SM, Beckmann CF (2006) Consistent resting-state networks across healthy subjects. Proc Natl Acad Sci U S A 103:13848-13853.

Dirksen CL, Howard JA, Cronin-Golomb A, Oscar-Berman M (2006) Patterns of prefrontal dysfunction in alcoholics with and without Korsakoff's syndrome, patients with Parkinson's disease, and patients with rupture and repair of the anterior communicating artery. Neuropsychiatr Dis Treat 2:327-339.

Everitt BJ, Robbins TW (2005) Neural systems of reinforcement for drug addiction: from actions to habits to compulsion. Nat Neurosci 8:14811489.

Fair DA, Dosenbach NUF, Church JA, Cohen AL, Brahmbhatt S, Miezin FM, Barch DM, Raichle ME, Petersen SE, Schlaggar BL (2007) Development of distinct control networks through segregation and integration. Proc Natl Acad Sci U S A 104:13507-13512.

Goldstein RZ, Leskovjan AC, Hoff AL, Hitzemann R, Bashan F, Khalsa SS, Wang G-J, Fowler JS, Volkow ND (2004) Severity of neuropsychological impairment in cocaine and alcohol addiction: association with metabolism in the prefrontal cortex. Neuropsychologia 42:1447-1458.

Greicius MD, Krasnow B, Reiss AL, Menon V (2003) Functional connectivity in the resting brain: a network analysis of the default mode hypothesis. Proc Natl Acad Sci U S A 100:253-258. 
Gu H, Salmeron BJ, Ross TJ, Geng X, Zhan W, Stein EA, Yang Y (2010) Mesocorticolimbic circuits are impaired in chronic cocaine users as demonstrated by resting-state functional connectivity. NeuroImage 53:593-601.

Hampson M, Driesen NR, Skudlarski P, Gore JC, Constable RT (2006) Brain connectivity related to working memory performance. J Neurosci 26:13338-13343.

Harding AJ, Halliday GM, Ng JLF, Harper CG, Kril JJ (1996) Loss of vasopressin-immunoreactive neurons in alcoholics is dose-related and timedependent. Neuroscience 72:699-708.

Heather N, Booth P, Luce A (1998) Impaired Control Scale: cross-validation and relationships with treatment outcome. Addiction 93:761-771.

Kiviniemi V, Starck T, Remes J, Long X, Nikkinen J, Haapea M, Veijola J, Moilanen I, Isohanni M, Zang Y-F, Tervonen O (2009) Functional segmentation of the brain cortex using high model order group PICA. Hum Brain Mapp 30:3865-3886.

Kril JJ, Halliday GM, Svoboda MD, Cartwright H (1997) The cerebral cortex is damaged in chronic alcoholics. Neuroscience 79:983-998.

Krmpotich TD, Tregellas JR, Thompson LL, Banich MT, Klenk AM, Tanabe JL (2013) Resting-state activity in the left executive control network is associated with behavioral approach and is increased in substance dependence. Drug Alcohol Depend 129:1-7.

Laird AR, Fox PM, Eickhoff SB, Turner JA, Ray KL, McKay DR, Glahn DC, Beckmann CF, Smith SM, Fox PT (2011) Behavioral interpretations of intrinsic connectivity networks. J Cogn Neurosci 23:4022-4037.

Lishman WA (1990) Alcohol and the brain. Br J Psychiatry 156:635-644.

Lynall M-E, Bassett DS, Kerwin R, McKenna PJ, Kitzbichler M, Muller U, Bullmore E (2010) Functional connectivity and brain networks in schizophrenia. J Neurosci 30:9477-9487.

Lyvers M (2000) "Loss of control" in alcoholism and drug addiction: a neuroscientific interpretation. Exp Clin Psychopharmacol 8:225-245.

MacCallum RC, Wegener DT, Uchino BN, Fabrigar LR (1993) The problem of equivalent models in applications of covariance structure analysis. Psychol Bull 114:185-199.

Miyake A, Friedman NP, Emerson MJ, Witzki AH, Howerter A, Wager TD (2000) The unity and diversity of executive functions and their contributions to complex "frontal lobe" tasks: a latent variable analysis. Cogn Psychol 41:49-100.

Moselhy HF, Georgiou G, Kahn A (2001) Frontal lobe changes in alcoholism: a review of the literature. Alcohol Alcohol 36:357-368.

Oscar-Berman M, Shagrin B, Evert DL, Epstein C (1997) Impairments of brain and behavior: the neurological effects of alcohol. Alcohol Health Res World 21:65-75.

Park SQ, Kahnt T, Beck A, Cohen MX, Dolan RJ, Wrase J, Heinz A (2010) Prefrontal cortex fails to learn from reward prediction errors in alcohol dependence. J Neurosci 30:7749-7753.

Parsons OA, Nixon SJ (1993) Neurobehavioral sequelae of alcoholism. Neurol Clin 11:205-218.

Pfefferbaum A, Rosenbloom M, Rohlfing T, Sullivan EV (2009) Degradation of association and projection white matter systems in alcoholism detected with quantitative fiber tracking. Biol Psychiatry 65:680-690.

Pfefferbaum A, Sullivan EV, Mathalon DH, Lim KO (1997) Frontal lobe volume loss observed with magnetic resonance imaging in older chronic alcoholics. Alcohol Clin Exp Res 21:521-529.

Pitel AL, Beaunieux H, Witkowski T, Vabret F, Guillery-Girard B, Quinette P, Desgranges B, Eustache F (2007) Genuine episodic memory deficits and executive dysfunctions in alcoholic subjects early in abstinence. Alcohol Clin Exp Res 31:1169-1178.

Power JD, Barnes KA, Snyder AZ, Schlaggar BL, Petersen SE (2012) Spurious but systematic correlations in functional connectivity MRI networks arise from subject motion. NeuroImage 59:2142-2154.

Rogers BP, Parks MH, Nickel MK, Katwal SB, Martin PR (2012) Reduced fronto-cerebellar functional connectivity in chronic alcoholic patients. Alcohol Clin Exp Res 36:294-301.

Saunders JB, Aasland OG, Babor TF, De La Fuente JR, Grant M (1993) Development of the alcohol use disorders identification test (AUDIT): WHO collaborative project on early detection of persons with harmful alcohol consumption-II. Addiction 88:791-804.
Schulte T, Muller-Oehring EM, Pfefferbaum A, Sullivan EV (2010) Neurocircuitry of emotion and cognition in alcoholism: contributions from white matter fiber tractography. Dialogues Clin Neurosci 12:554-560.

Scott A, Courtney W, Wood D, de la Garza R, Lane S, King M, Wang R, Roberts J, Turner JA, Calhoun VD (2011) COINS: an innovative informatics and neuroimaging tool suite built for large heterogeneous datasets. Front Neuroinform 5:33.

Seeley WW, Menon V, Schatzberg AF, Keller J, Glover GH, Kenna H, Reiss AL, Greicius MD (2007) Dissociable intrinsic connectivity networks for salience processing and executive control. J Neurosci 27:2349-2356.

Shirer WR, Ryali S, Rykhlevskaia E, Menon V, Greicius MD (2012) Decoding subject-driven cognitive states with whole-brain connectivity patterns. Cereb Cortex 22:158-165.

Singh-Curry V, Husain M (2009) The functional role of the inferior parietal lobe in the dorsal and ventral stream dichotomy. Neuropsychologia 47:1434-1448.

Smith S, Fein G (2010) Cognitive performance in treatment-naïve active alcoholics. Alcohol Clin Exp Res 34:2097-2105.

Smith SM, Fox PT, Miller KL, Glahn DC, Fox PM, Mackay CE, Filippini N, Watkins KE, Toro R, Laird AR, Beckmann CF (2009) Correspondence of the brain's functional architecture during activation and rest. Proc Natl Acad Sci U S A 106:13040-13045.

Sullivan E, Pfefferbaum A (2005) Neurocircuitry in alcoholism: a substrate of disruption and repair. Psychopharmacology 180:583-594.

Sullivan EV, Rosenbloom MJ, Pfefferbaum A (2000) Pattern of motor and cognitive deficits in detoxified alcoholic men. Alcohol Clin Exp Res 24:611-621.

Tomasi D, Volkow ND (2012) Aging and functional brain networks. Mol Psychiatry 17:549-558.

Upadhyay J, Maleki N, Potter J, Elman I, Rudrauf D, Knudsen J, Wallin D, Pendse G, McDonald L, Griffin M, Anderson J, Nutile L, Renshaw P, Weiss R, Becerra L, Borsook D (2010) Alterations in brain structure and functional connectivity in prescription opioid-dependent patients. Brain 133:2098-2114.

Van Dijk KRA, Sabuncu MR, Buckner RL (2012) The influence of head motion on intrinsic functional connectivity MRI. NeuroImage 59:431-438.

Welsh RC, Chen AC, Taylor SF (2010) Low-frequency BOLD fluctuations demonstrate altered thalamocortical connectivity in schizophrenia. Schizophr Bull 36:713-722.

Wig GS, Schlaggar BL, Petersen SE (2011) Concepts and principles in the analysis of brain networks. Ann N Y Acad Sci 1224:126-146.

\section{SUPPORTING INFORMATION}

Additional Supporting Information may be found in the online version of this article:

Data S1. Methods and results.

Figure S1. Regions with functional connectivity to the seed region in left parietal lobe (blue) representing the left executive control network. Color bar represents $t$-scores.

Figure S2. Regions with functional connectivity to the seed region in bilateral thalami (blue) representing the basal ganglia network.

Figure S3. Regions with functional connectivity to the seed region in supplementary motor area (blue) representing the sensorimotor network.

Figure S4. Regions with functional connectivity to the seed region in V1 (blue) representing the primary visual network.

Figure S5. Regions with functional connectivity to the seed region in left parietal lobe (blue), which were negatively correlated with failure to control alcohol consumption. 